\title{
Dexmedetomidine activates the PI3K/Akt pathway to inhibit hepatocyte apoptosis in rats with obstructive jaundice
}

\author{
YAYING XIE*, CHUNYAN GUO*, YE LIU, LUANYUAN SHI and JIANSHE YU \\ Department of Anesthesiology, The Affiliated Hospital of Inner Mongolia Medical University, \\ Hohhot, Inner Mongolia Autonomous Region 010050, P.R. China
}

Received June 3, 2018; Accepted March 28, 2019

DOI: $10.3892 /$ etm.2019.8085

\begin{abstract}
Obstructive jaundice (OJ) is a common disease in clinical surgery. The present study aimed to determine the effects of dexmedetomidine (Dex) on hepatocyte apoptosis in rats with $\mathrm{OJ}$ and also to explore the underlying mechanism. A total of 30 adult male Sprague Dawley rats were randomly divided into 3 groups: Sham group, bile duct ligation (BDL) group, and BDL+Dex group. The serum liver function index, expression levels of serum inflammatory factor interleukin-6 (IL-6) and tumor necrosis factor- $\alpha$ (TNF- $\alpha$ ), and the liver pathological changes were compared amongst groups. The serum liver function index and expression levels of inflammatory factors in the BDL group and BDL+Dex group were higher compared with the sham group. The serum liver function index and expression levels of inflammatory factors were lower in the BDL+Dex group compared with the BDL group. The severity of hepatic injury was diminished in the BDL+Dex group compared with the BDL group. Compared with the sham group, the hepatocyte apoptosis rate increased significantly in the BDL group and BDL+Dex group. The present findings suggested that Dex improved the liver function of rats with $\mathrm{OJ}$, reduced the production of inflammatory factors and inhibited the apoptosis of hepatocytes. Dex demonstrated a protective effect on liver damage potentially via activation of the phosphoinositide 3-kinase/protein kinase B signaling pathway.
\end{abstract}

Correspondence to: Professor Jianshe Yu, Department of Anesthesiology, The Affiliated Hospital of Inner Mongolia Medical University, 1 Tongdao North Street, Huimin, Hohhot, Inner Mongolia Autonomous Region 010050, P.R. China

E-mail: yujianshe15c3@163.com

*Contributed equally

Key words: dexmedetomidine, obstructive jaundice, apoptosis, phosphoinositide 3-kinase/protein kinase B signaling pathway, liver protection

\section{Introduction}

Obstructive jaundice (OJ) is a common disease in clinical surgery. It can cause damage to various tissues and organs in the body with the most serious leading to hepatic injury. Studies have demonstrated that the accumulation of inflammatory factors in liver tissues and abnormal serum biochemical indexes are important causes of hepatic injury (1-3). However, the specific mechanism of hepatic injury caused by OJ remains not fully elucidated. An abnormal increase in hepatocyte apoptosis is currently considered to be the pathogenesis of hepatic atrophy caused by $\mathrm{OJ}(4,5)$. It has been reported that, due to endotoxin stimulation during OJ, Kupffer cells secrete a large number of inflammatory factors, such as tumor necrosis factor- $\alpha$ (TNF- $\alpha$ ) and interleukin-6 (IL-6), which induce hepatocyte apoptosis $(6,7)$. Hepatocyte apoptosis is a complex process involving multiple factors. Therefore, it is of great significance to better understand the mechanism of hepatocyte apoptosis for the prevention and treatment of hepatic injury caused by OJ.

Dexmedetomidine (Dex) is a new type of highly selective adrenergic receptor agonist that has been widely used in the clinic. Its molecular formula is $\mathrm{C}_{13} \mathrm{H}_{16} \mathrm{~N}_{2}$ containing methyl and imidazole functional groups. Dex can bind the presynaptic $\alpha_{2}$ adrenoceptor, activate the negative feedback loop of the sympathetic response and reduce the release of norepinephrine, thereby inhibiting the sympathetic reflex and stress response, and maintaining homeostasis (8). It has been demonstrated that Dex can inhibit the inflammatory reaction induced by endotoxemia whilst also having a protective effect on organ tissues (9). Zhang et al (10) reported that Dex inhibited the release of inflammatory cytokines such as IL- 6 and TNF- $\alpha$, and alleviated local and systemic inflammatory reactions. Dex protects the lung via increasing the expression levels of protein kinase B (Akt) in acute lung injury tissues (11). The phosphoinositide 3-kinase (PI3K)/Akt signaling pathway is widely present in cells, and is also one of the important pathways involved in the regulation of cell apoptosis. However, to the best of our knowledge, the effect and mechanism of Dex on hepatocyte apoptosis in an OJ rat model has not been reported. Therefore, the present study investigated an OJ rat model treated with Dex to observe the liver tissue inflammatory reaction and hepatocyte apoptosis. The present findings provided the theoretical and experimental basis for Dex in treating hepatic injury caused by OJ. 


\section{Materials and methods}

Experimental animals. A total of 30 healthy male 8-week-old Sprague Dawley (SD) rats (Shanghai SLAC Laboratory Animal Co., Ltd.) weighing $250 \pm 20 \mathrm{~g}$ each were raised in the Animal Experimental Center of the Affiliated Hospital of Inner Mongolia Medical University. The temperature of the housing area was $21 \pm 2^{\circ} \mathrm{C}$ with a relative humidity of $30-70 \%$ and light-dark cycle of 12/12 h. Rats were fed three times a day. Rats were fasted for $12 \mathrm{~h}$ before surgery and had free access to water. The Animal Experimental Ethics Committee of the Affiliated Hospital of Inner Mongolia Medical University approved this research.

Establishment of the OJ rat model. All rats were fasted for $12 \mathrm{~h}$ prior to surgery with free access to water. The rats were anesthetized by intraperitoneal injection of $2 \%$ sodium pentobarbital (30 mg/kg). The abdominal cavity was ascended through the midline incision under aseptic operations, and the bile duct was found in the hepatoduodenal ligament. The bile duct was double ligated with 5-0 silk thread at a distance of $0.8 \mathrm{~cm}$ from the hilum, and the incision was sutured layer by layer.

Experimental grouping. The SD rats were randomly divided into 3 groups: Sham group, bile duct ligation (BDL) group and $\mathrm{BDL}+\mathrm{Dex}$ group, with 10 rats in each group. In the sham group, the bile ducts of the rats were isolated only and BDL was not implemented. The rats in BDL group underwent surgery to establish the OJ model. After successful construction, the rats were injected with saline via the tail vein. The rats in BDL+Dex group underwent surgery to establish the OJ model. After successful construction, the rats were injected with $100 \mu \mathrm{g} / \mathrm{kg}$ Dex via the tail vein once a day. After 1 week of intervention, the SD rats were anesthetized with intraperitoneal injection of $2 \%$ pentobarbital $(30 \mathrm{mg} / \mathrm{kg})$, and then sacrificed by spinal dislocations for subsequent experimentation.

Observation indexes. The serum liver function indexes, IL-6 and TNF- $\alpha$ expression levels, liver pathological changes, hepatocyte apoptosis rate, Akt expression levels, phosphorylation of Akt (p-Akt; Thr308), caspase-3 and cleaved-caspase-3 protein expression in liver tissues were compared among groups.

Serum liver function indexes. Following 1 week of intervention, $2 \mathrm{ml}$ of blood was taken from the tail vein of rats from each group, and the serum was separated via centrifugation at $1,500 \mathrm{x} \mathrm{g}$ for $10 \mathrm{~min}$. The indexes of alanine aminotransferase (ALT), aspartate aminotransferase (AST) and total bilirubin (TBIL) were detected by iMagic automatic biochemical analyzer (China Shenzhen Kubel Biotechnology Co., Ltd.). Data were analyzed by iMagic Manager (version 1.0; China Shenzhen Kubel Biotechnology Co., Ltd.).

Expression levels of serum inflammatory cytokines IL- 6 and TNF- $\alpha$ measured with ELISA. Following 1 week of intervention, $2 \mathrm{ml}$ of blood was taken from the tail vein of rats from each group. The serum was separated from the blood by centrifuging the blood at $4^{\circ} \mathrm{C}$ and $1,000 \mathrm{xg}$ for $5 \mathrm{~min}$. The contents of IL-6 (cat. no. PR6000B) and TNF- $\alpha$ (cat. no. PRTA00) were detected by enzyme-linked immunosorbent assay (ELISA) supplied by R\&D Systems, Inc. and the operation procedure was strictly in accordance with the manufacturer's protocol.

Liver pathological changes following immunostaining. Following anesthesia, the livers from each group of rats were exposed along the original incision, then part of the liver tissue was removed and quickly stored in liquid nitrogen for later use. A selected portion of liver tissues was fixed with $4 \%$ paraformaldehyde solution at $4^{\circ} \mathrm{C}$ for $12 \mathrm{~h}$, and routine hematoxylin and eosin staining was applied after paraffin-embedded $5-\mu$ m-thick section. The sections were stained with hematoxylin for $3 \mathrm{~min}$ and eosin for $2 \mathrm{~min}$ at room temperature. The pathological structure of the liver tissues of each group was examined under a light microscope (magnification, x200).

Hepatocyte apoptosis rate using flow cytometry. Under aseptic operation, the shredded liver tissues were completely digested in $0.3 \%$ type I collagenase digestive solution. The cells were then collected into a centrifuge tube using a $200 \mu \mathrm{m}$ cell strainer and prepared into a single cell suspension with the concentration adjusted to $1 \times 10^{6} \mathrm{cell} / \mathrm{ml}$. The cell suspension of $100 \mu \mathrm{l}$ was collected and $5 \mu \mathrm{l}$ Annexin V-fluorescein isothiocyanate and $10 \mu \mathrm{l}$ propidium iodide solution from FITC Annexin V Apoptosis Detection Kit I (cat. no. 556547; both BD Biosciences) were added. It was mixed evenly then incubated at room temperature for $15 \mathrm{~min}$ in the dark. Then $400 \mu \mathrm{l}$ of PBS was added into the mixture, and the rate of hepatocyte apoptosis was measured via FACS Canto II flow cytometry (BD Biosciences). The hepatocyte apoptosis rate in each group was analyzed by Accuri ${ }^{\text {TM }}$ C6 software (version 6.2; BD Biosciences).

Expression levels of Akt, p-Akt (Thr308), caspase-3 and cleaved-caspase-3 protein in liver tissues measured by western blot analysis. The liver tissues were ground and lysed, and total protein was extracted from the liver tissues collected from each group of rats with the RIPA buffer (Sigma-Aldrich) containing $1 \%$ sodium deoxycholate and $0.1 \%$ SDS. The protein concentration was determined using the bicinchoninic acid protein concentration assay kit (cat. no. P0012-1; Beyotime Institute of Biotechnology). The protein samples were separated liver tissues were ground and lysed, Then electrophoresis separation was performed on protein samples (30 $\mu \mathrm{g} /$ lane) using SDS-PAGE gel (5\% spacer gel and 10\% separation gel). The protein was transferred to polyvinylidene fluoride membrane via the enzyme-linked immunoelectrotransfer blot method. The membranes were blocked using Tris-buffered saline-T solution containing 5\% skimmed milk powder at room temperature for $2 \mathrm{~h}$ then incubated with primary antibodies against Akt (1:400; cat. no. sc-5298; Santa Cruz Biotechnology, Inc.), p-Akt (1:500; cat. no. sc-33437; Santa Cruz Biotechnology, Inc.), cleaved-caspase-3 (1:700; cat. no. 9661S Cell Signaling Technology, Inc.), caspase-3 (1:500; cat. no. sc-271759; Santa Cruz Biotechnology, Inc.) overnight at $4^{\circ} \mathrm{C}$. Following washes with TBST, the membrane was incubated with a horseradish peroxidase-labeled goat anti-rabbit immunoglobulin $\mathrm{G}$ secondary antibody (1:600; cat. no. A0208; Beyotime Institute of Biotechnology) and incubated at $4{ }^{\circ} \mathrm{C}$ for $2 \mathrm{~h}$; then the membrane was visualized using hypersensitive electrogenerated chemiluminescence reagent (Beyotime Institute of Biotechnology). ImageJ software (version 1.8.0; National 
Table I. Comparison of liver function in each group.

\begin{tabular}{lccc}
\hline Group & ALT $(\mathrm{U} / \mathrm{l})$ & AST $(\mathrm{U} / \mathrm{l})$ & TBIL $(\mu \mathrm{mol} / \mathrm{l})$ \\
\hline Sham group & $43.92 \pm 17.56$ & $138.72 \pm 32.57$ & $11.24 \pm 2.43$ \\
BDL group & $794.46 \pm 118.23^{\mathrm{a}}$ & $1,358.61 \pm 225.86^{\mathrm{a}}$ & $119.25 \pm 20.18^{\mathrm{a}}$ \\
BDL+Dex group & $271.31 \pm 97.85^{\mathrm{a}, \mathrm{b}}$ & $537.91 \pm 180.42^{\mathrm{a}, \mathrm{b}}$ & $64.07 \pm 12.29^{\mathrm{a}, \mathrm{b}}$ \\
F-test & 25.063 & 28.725 & 31.284 \\
P-value & 0.000 & 0.000 & 0.000 \\
\hline
\end{tabular}

${ }^{\mathrm{a}} \mathrm{P}<0.001$ vs. sham group; ${ }^{\mathrm{b}} \mathrm{P}<0.001$ vs. BDL group. Dex, dexmedetomidine; BDL, bile duct ligation; ALT, alanine aminotransferase; AST, aspartate aminotransferase; TBIL, total bilirubin.
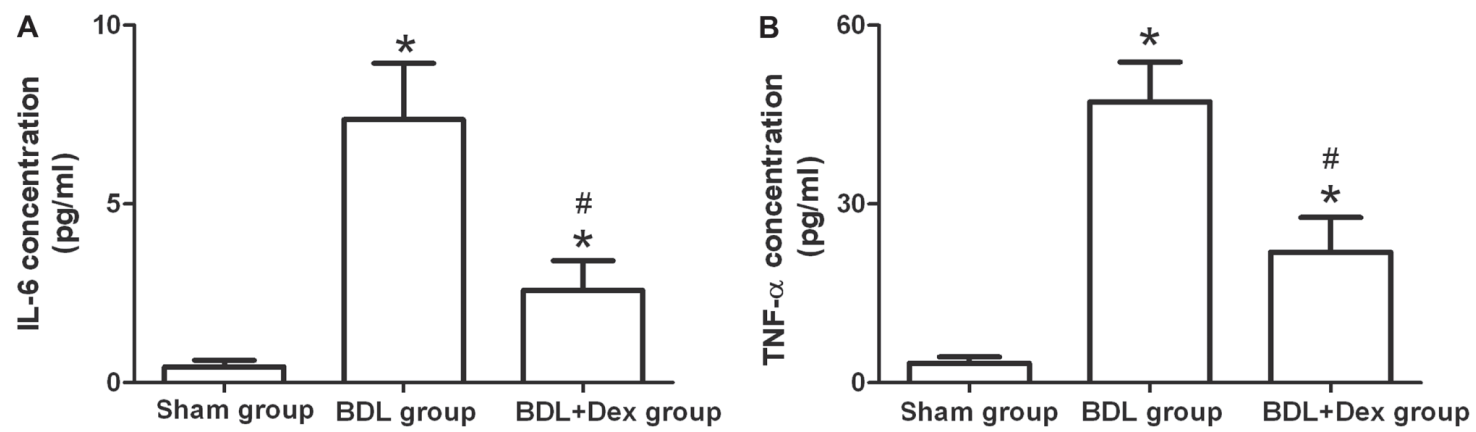

Figure 1. Dex treatment significantly reduces inflammatory cytokine expression following BDL. (A) IL-6 expression levels and (B) TNF- $\alpha$ expression levels measured using ELISA. "P<0.05 vs. sham group; ${ }^{\#} \mathrm{P}<0.05$ vs. BDL group. Dex, dexmedetomidine; BDL, bile duct ligation; IL-6, interleukin-6; TNF- $\alpha$, tumor necrosis factor- $\alpha$.

Institutes of Health) was used for quantitative analysis of the bands. GAPDH was used as an internal reference to compare the optical density values of the target bands in each group.

Statistical analysis. All data were analyzed using SPSS v.20.0 statistical software (IBM Corp.) The count data were expressed as a percentage and the chi-square test was used for comparisons amongst groups. The measurement data were expressed as mean \pm standard deviation. The comparison between two groups was performed by independent sample t-test, and the comparison of three groups was analyzed by one-way analysis of variance with Bonferroni method as post hoc test. $\mathrm{P}<0.05$ was considered to indicate statistical significance.

\section{Results}

Dex treatment decreases the serum levels of ALT, AST and TBIL following BDL. Compared with the sham group, the serum levels of ALT, AST and TBIL in the BDL and BDL+Dex group increased significantly $(\mathrm{P}<0.001$; Table I). However, compared with the BDL group, the serum levels of ALT, AST and TBIL in the BDL+Dex group decreased significantly $(\mathrm{P}<0.001$; Table I).

Dex treatment decreases IL- 6 and TNF- $\alpha$ levels. The expression levels of serum IL- 6 and TNF- $\alpha$ in the rats of the BDL+Dex group were significantly lower compared with the BDL group (P<0.05; Fig. 1). The expression levels of IL-6 and TNF- $\alpha$ in rats of the BDL group and the BDL+Dex group were significantly higher compared with the sham group $(\mathrm{P}<0.05$; Fig. 1$)$.
Dex treatment attenuates liver pathological changes following $B D L$. Optical microscopy demonstrated that there were no abnormal changes in the morphology of hepatocytes in the sham group with the structures of the hepatic lobule, portal area and central venous well defined (Fig. 2A). The hepatocytes of BDL group were significantly degenerated and necrotic with the hepatic lobule structure damaged and pseudo-lobule formed. Inflammatory cells had also infiltrated the structure (Fig. 2B). The liver pathological changes in the BDL+Dex group were intermediate between the aforementioned two groups, as the damage to hepatocytes was improved, the arrangement of the hepatic cord was relatively neat and the structure of hepatic lobule was relatively clear. Only a small number of inflammatory cells had infiltrated the structure (Fig. 2C).

Dex treatment decreases apoptosis rate of hepatocytes following $B D L$. Flow cytometry demonstrated that the apoptosis rate of hepatocytes in the sham group was $3.25 \pm 0.54 \%$. Compared with the sham group, the apoptosis rate of hepatocytes in the BDL group and BDL+Dex group increased significantly $(\mathrm{P}<0.05$; Fig. $3 \mathrm{~A}-\mathrm{C})$. However, compared with the BDL group $(29.54 \pm 1.48 \%)$, the apoptosis rate of hepatocytes in the BDL+Dex group $(16.24 \pm 1.18 \%)$ decreased significantly $(\mathrm{P}<0.05$; Fig. 3).

Comparisons of Akt, p-Akt, caspase-3 and cleaved caspase-3 protein expression in liver tissues of rats. The levels of Akt, caspase- 3 and cleaved caspase- 3 in each group were expressed relative to the GAPDH control, while the levels of $\mathrm{p}$-Akt in each group were calculated relative to the total 

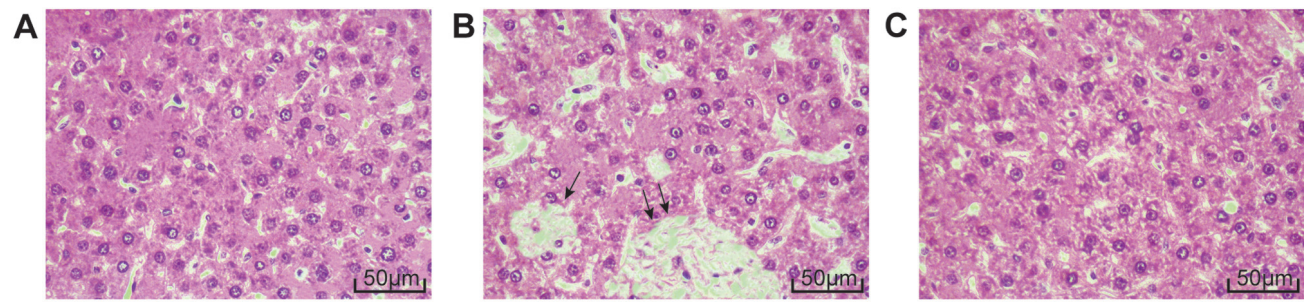

Figure 2. Dex treatment attenuates liver damage following BDL. (A) Hematoxylin and eosin staining of liver tissue specimens in the sham group demonstrated no abnormal changes in morphology of hepatocytes, and the structures of hepatic lobules, portal areas, and central veins were clear. (B) The hepatocytes of rats in the BDL group were significantly degenerated and necrotic, with the hepatic lobule structure damaged. A pseudo-lobule (single arrow), and infiltrated inflammatory cells (double arrow) are highlighted. (C) The damage to hepatocytes in the BDL+Dex group was significantly improved, and hepatic cord arrangement was relatively neat. The structure of the hepatic lobule was relatively clear, and only a small amount of inflammatory cells had infiltrated Dex, dexmedetomidine; BDL, bile duct ligation.
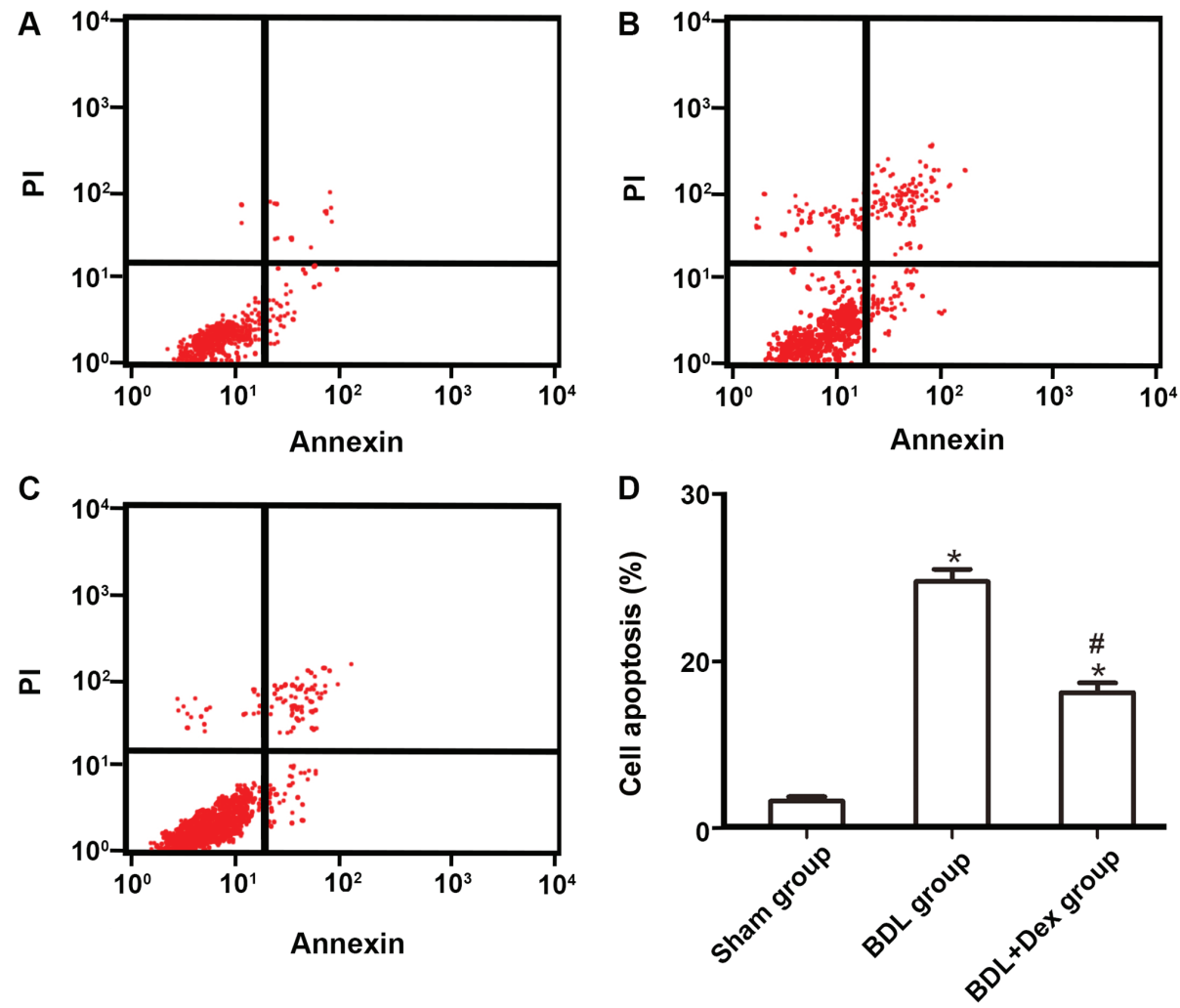

Figure 3. Dex treatment reduces rate of hepatocyte apoptosis following BDL. (A) Apoptosis rate of hepatocytes analyzed using flow cytometry in (A) sham group, (B) BDL group and (C) BDL+Dex group. (D) Comparison of apoptosis rate in each group. " $\mathrm{P}<0.05$ vs. sham group; "P<0.05 vs. BDL group. Dex, dexmedetomidine; BDL, bile duct ligation; PI, propidium iodide.

Akt. Compared with the sham group, Akt and p-Akt expression decreased in the BDL and BDL+Dex group, whilst the expression of caspase- 3 protein decreased significantly and the expression of cleaved caspase-3 protein increased significantly $(\mathrm{P}<0.05$; Fig. 4). Compared with the BDL group, the Akt and p-Akt expressions in the BDL+Dex group increased, whilst the expression of caspase-3 protein increased significantly and levels of cleaved caspase- 3 protein decreased significantly $(\mathrm{P}<0.05$; Fig. 4).

\section{Discussion}

$\mathrm{OJ}$ is a common pathogenesis of many bile duct diseases and a relatively prevalent surgical disease; however, its aetiology is complicated. OJ causes accumulation of inflammatory factors such as TNF- $\alpha$ and IL-6 in liver tissues, and also infiltration of inflammatory cells such as neutrophils. This results in elevated hepatic function markers such as AST, ALT and TBIL, eventually leading to hepatic parenchyma injury and a decrease in hepatocytes $(12,13)$. It has been demonstrated that a decrease of hepatocytes during OJ development is mainly caused by apoptosis due to cholestasis, mitochondrial dysfunction, reactive oxygen species and certain cytokines that can affect the process of apoptosis, but the specific mechanism is not clear (14-16). Therefore, relieving biliary obstruction, alleviating the inflammatory reaction and inhibiting hepatocyte apoptosis are key to reducing hepatic damage in patients.

Dex is a novel highly selective adrenergic receptor agonist that displays strong intrinsic activity, short elimination half-life and distribution half-life (17). Recent studies have 
A

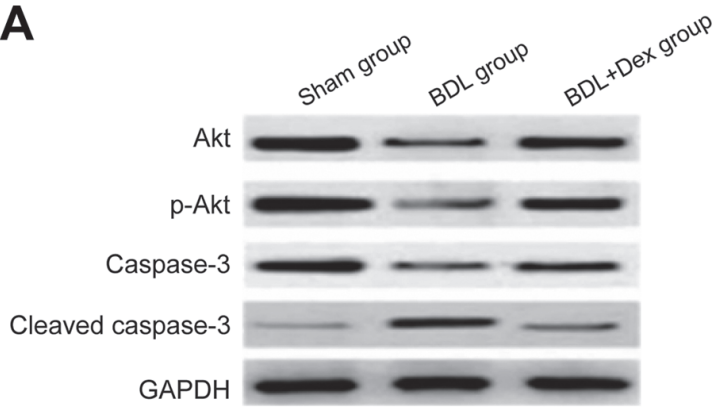

C

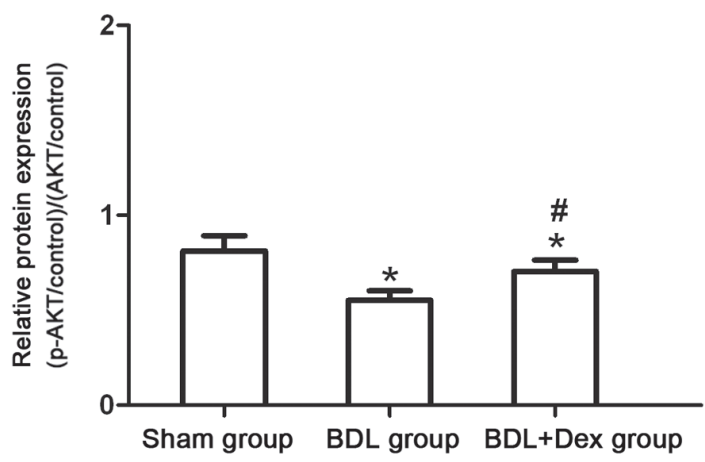

B
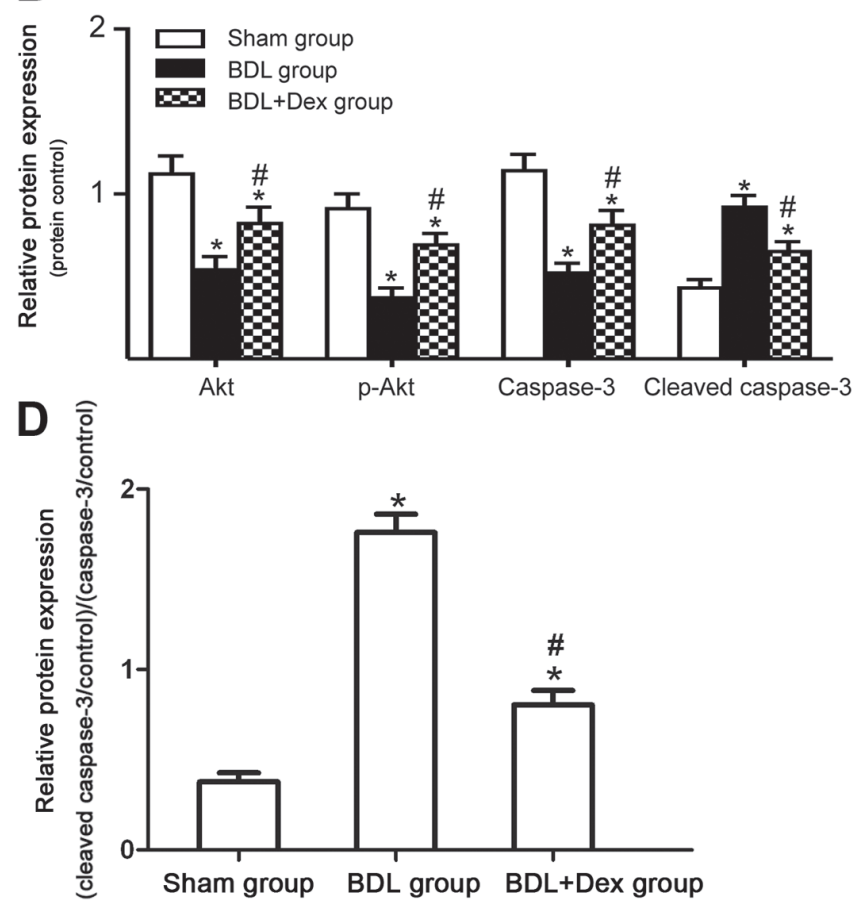

Figure 4. Expression of Akt, p-Akt, caspase-3 and cleaved caspase-3 proteins in liver tissues of each group. (A) Representative western blots. (B) Quantification of protein levels relative to GAPDH. (C) Relative expression levels of post-translational modifications of p-Akt. (D) Relative expression levels of post-translational modifications of cleaved caspase-3. "P $<0.05$ vs. sham; ${ }^{\#} \mathrm{P}<0.05$ vs. BDL group. Akt, protein kinase B; p-Akt, phosphorylation of protein kinase B; Dex, dexmedetomidine; BDL, bile duct ligation.

demonstrated that Dex has protective effects on the heart, kidney, central nervous system, respiratory system and small intestine $(18,19)$. Presently, the protective effects of Dex on hepatic injury have received increased attention. It has been reported that Dex can significantly stabilize hemodynamics and improve the quality of recovery in patients with OJ during anesthesia (20). Research into the effects of Dex in OJ is mainly focused on animal experimentation. For example, it has been identified that intraperitoneal injection of Dex can significantly reduce the oxidative stress indexes in a rat model of hepatic ischemia-reperfusion whilst increasing the antioxidant capacity (21). Kucuk et al (22) reported that Dex could restore heat shock protein 60 and tumor protein p53 in hepatocytes of rats with ischemia-reperfusion injury and protect the liver from injury by regulating the expression of apoptosis-related proteins. The results of the present study demonstrated that Dex could not only significantly improve the pathological morphology of liver tissues and the changes of hepatic function parameters ALT, AST and TBIL, but also inhibit the expression levels of serum inflammatory cytokines IL- 6 and TNF- $\alpha$ in rats with OJ. The present findings indicated that Dex had a protective effect on hepatic injury caused by OJ through reducing the levels of serum inflammatory cytokines IL-6 and TNF- $\alpha$, which is in agreement with the studies of Venn et al (23) and Wang et al (24).

Dex can exert cell survival effects by activating the PI3K/Akt signaling pathway (25). The caspase enzyme family is one of the important downstream effectors of the PI3K/Akt pathway. The process of apoptosis involves the cascade amplification reaction process of irreversible limited hydrolytic substrate of caspases, with caspase-3 the most critical downstream apoptotic protease. It has been reported that the activation of PI3K/Akt leads to the phosphorylation of the proapoptotic protein Bcl-2-associated death promoter in nerve cells, the upregulation of the Inhibitior of apoptotis proteins levels, and the inhibition of the endogenous apoptotic pathway regulated by the Caspase cascade signal pathway, eventually exerting a cell protective effect (26). A study has demonstrated that $100 \mathrm{ng} / \mathrm{ml}$ Dex can induce apoptosis of inflammatory cells by activating the caspase cascade reaction of neutrophils and other inflammatory cells, whilst $1 \mathrm{ng} / \mathrm{ml}$ Dex has no significant effect on apoptosis of neutrophils (27), indicating that Dex has dose dependent effects on cell apoptosis. The results of the present study determined that compared with the BDL group, the hepatocyte apoptosis rate in the OJ rat model following Dex treatment was significantly lower. The expression levels of Akt and p-Akt in liver tissues of the BDL+Dex group were significantly increased, the expression of caspase-3 protein was significantly higher, and the expression levels of cleaved caspase-3 were significantly lower compared with the OJ rat model group. Findings suggested that Dex activated the PI3K/Akt signaling pathway, inhibited the activation level of caspase-3, and reduced the apoptosis of hepatocytes.

In summary, OJ induced hepatocyte apoptosis in rats. Dex significantly improved the OJ-induced injury of hepatic tissues and reduced hepatocyte apoptosis. It was hypothesized that Dex had a protective role potentially via activating the PI3K/Akt signaling pathway.

\section{Acknowledgements}

Not applicable. 


\section{Funding}

This work was supported by the Medical and Health Research Project of the Health and Family Planning Commission of Inner Mongolia Autonomous Region (grant. no. 201701070), and Fund Project of Inner Mongolia Science and Technology Department [grant. no. 2015MS(LH)0809].

\section{Availability of data and materials}

The datasets used and/or analyzed during the current study are available from the corresponding author on reasonable request.

\section{Authors' contributions}

YX was the guarantor for the integrity of the study and contributed to study concepts, study design, definition of intellectual content, statistical analysis, manuscript preparation and manuscript editing. CG collected and analyzed the data, prepared the figures and also contributed substantially to manuscript revision. CG and LS were responsible for the production of animal models of obstructive jaundice. YL and LS drafted the manuscript. YL also collated and analyzed the current literature on this subject, and was responsible for the breeding and laboratory work of rats. LS constructed the animal models of obstructive jaundice and collected the data.

\section{Ethics approval and consent to participate}

The present study was approved by the Ethics Committee of the Affiliated Hospital of Inner Mongolia Medical University (Inner Mongolia, China).

\section{Patient consent for publication}

Not applicable.

\section{Competing interests}

The authors declare that they have no competing interests.

\section{References}

1. Li X, Li J, Ou YJ, Zhu XX, Yin XY, Zhu YX and Tang D: Hepatoprotective effect of ulinastatin in a rat model of major hepatectomy after obstructive jaundice. Dig Dis Sci 60: 1680-1689, 2015.

2. Pavlidis ET and Pavlidis TE: Pathophysiological consequences of obstructive jaundice and perioperative management Hepatobiliary Pancreat Dis Int 17: 17-21, 2018.

3. Unal Y, Tuncal S, Kosmaz K, Kucuk B, Kismet K, Cavusoglu T, Celepli P, Senes M, Yildiz S and Hucumenoglu S: The effect of calcium dobesilate on liver damage in experimental obstructive jaundice. J Invest Surg 32: 238-244, 2019.

4. Liu Q, Li BS, Song YJ, Hu MG, Lu JY, Gao A, Sun XJ, Guo XM and Liu R: Hydrogen-rich saline protects against mitochondrial dysfunction and apoptosis in mice with obstructive jaundice. Mol Med Rep 13: 3588-3596, 2016.

5. Fang D, He Y and Luan Z: Simvastatin augments activation of liver regeneration through attenuating transforming growth factor-betal induced-apoptosis in obstructive jaundice rats. Exp Ther Med 14: 4839-4845, 2017.

6. Monteiro MEL, Xavier AR and Azeredo VB: Diet and liver apoptosis in rats: A particular metabolic pathway. Nutr Hosp 34: 463-468, 2017.
7. Wang $\mathrm{H}$, Zhang $\mathrm{Y}$, Bai R, Wang $\mathrm{M}$ and Du S: Baicalin attenuates alcoholic liver injury through modulation of hepatic oxidative stress, Inflammation and Sonic hedgehog pathway in rats. Cell Physiol Biochem 39: 1129-1140, 2016.

8. Chen Z, Ding T and Ma CG: Dexmedetomidine (DEX) protects against hepatic ischemia/reperfusion (I/R) injury by suppressing inflammation and oxidative stress in NLRC5 deficient mice. Biochem Biophys Res Commun 493: 1143-1150, 2017.

9. Wang ZX, Huang CY, Hua YP, Huang WQ, Deng LH and Liu KX: Dexmedetomidine reduces intestinal and hepatic injury after hepatectomy with inflow occlusion under general anaesthesia: A randomized controlled trial. Br J Anaesth 112: 1055-1064, 2014.

10. Zhang X, Wang J, Qian W, Zhao J, Sun L, Qian Y and Xiao H: Dexmedetomidine inhibits tumor necrosis factor-alpha and interleukin-6 in lipopolysaccharide-stimulated astrocytes by suppression of c-Jun N-terminal kinases. Inflammation 37: 942-949, 2014.

11. Meng L, Li L, Lu S, Li K, Su Z, Wang Y, Fan X, Li X and Zhao G: The protective effect of dexmedetomidine on LPS-induced acute lung injury through the HMGB1-mediated TLR4/NF-kappaB and PI3K/Akt/mTOR pathways. Mol Immunol 94: 7-17, 2018.

12. Tag CG, Weiskirchen S, Hittatiya K, Tacke F, Tolba RH and Weiskirchen R: Induction of experimental obstructive cholestasis in mice. Lab Anim 49 (Suppl 1): S70-S80, 2015.

13. Taniguchi T, Kidani Y, Kanakura H, Takemoto Y and Yamamoto K: Effects of dexmedetomidine on mortality rate and inflammatory responses to endotoxin-induced shock in rats. Crit Care Med 32: 1322-1326, 2004.

14. Maillette de Buy Wenniger L and Beuers U: Bile salts and cholestasis. Dig Liver Dis 42: 409-418, 2010.

15. Sokol RJ, Dahl R, Devereaux MW, Yerushalmi B, Kobak GE and Gumpricht E: Human hepatic mitochondria generate reactive oxygen species and undergo the permeability transition in response to hydrophobic bile acids. J Pediatr Gastroenterol Nutr 41: 235-243, 2005.

16. Masud A, Mohapatra A, Lakhani SA, Ferrandino A, Hakem R and Flavell RA: Endoplasmic reticulum stress-induced death of mouse embryonic fibroblasts requires the intrinsic pathway of apoptosis. J Biol Chem 282: 14132-14139, 2007.

17. Lv M, Zeng H, He Y, Zhang J and Tan G: Dexmedetomidine promotes liver regeneration in mice after $70 \%$ partial hepatectomy by suppressing NLRP3 inflammasome not TLR4/NFkappaB. Int Immunopharmacol 54: 46-51, 2018.

18. Jiang L, Hu M, Lu Y, Cao Y, Chang Y and Dai Z: The protective effects of dexmedetomidine on ischemic brain injury: A meta-analysis. J Clin Anesth 40: 25-32, 2017.

19. Gong Z, Ma L, Zhong YL, Li J, Lv J and Xie YB: Myocardial protective effects of dexmedetomidine in patients undergoing cardiac surgery: A meta-analysis and systematic review. Exp Ther Med 13: 2355-2361, 2017.

20. Wang L and Yu WF: Obstructive jaundice and perioperative management. Acta Anaesthesiol Taiwan 52: 22-29, 2014.

21. Tufek A, Tokgoz O, Aliosmanoglu I, Alabalik U, Evliyaoglu O, Ciftci T, Guzel A and Yildirim ZB: The protective effects of dexmedetomidine on the liver and remote organs against hepatic ischemia reperfusion injury in rats. Int J Surg 11: 96-100, 2013.

22. Kucuk A, Yaylak F, Cavunt-Bayraktar A, Tosun M, Arslan M, Comu FM and Kavutcu M: The protective effects of dexmedetomidine on hepatic ischemia reperfusion injury. Bratisl Lek Listy 115: 680-684, 2014.

23. Venn RM, Bryant A, Hall GM and Grounds RM: Effects of dexmedetomidine on adrenocortical function, and the cardiovascular, endocrine and inflammatory responses in post-operative patients needing sedation in the intensive care unit. Br J Anaesth 86: 650-656, 2001.

24. Wang H,Hu B,Zou Y,BoL,Wang J,LiJ and Luo Y: Dexmedetomidine premedication attenuates concanavalin A-induced hepatitis in mice. J Toxicol Sci 39: 755-764, 2014.

25. Zhu YM, Wang CC, Chen L, Qian LB, Ma LL, Yu J, Zhu MH, Wen CY, Yu LN and Yan M: Both PI3K/Akt and ERK1/2 pathways participate in the protection by dexmedetomidine against transient focal cerebral ischemia/reperfusion injury in rats. Brain Res 1494: $1-8,2013$.

26. Jin XF, Wang S, Shen M, Wen X, Han XR, Wu JC, Tang GZ, Wu DM, $\mathrm{Lu} \mathrm{J}$ and Zheng YL: Effects of rehabilitation training on apoptosis of nerve cells and the recovery of neural and motor functions in rats with ischemic stroke through the PI3K/Akt and Nrf2/ARE signaling pathways. Brain Res Bull 134: 236-245, 2017.

27. Kishikawa H, Kobayashi K, Takemori K, Okabe T, Ito K and Sakamoto A: The effects of dexmedetomidine on human neutrophil apoptosis. Biomed Res 29: 189-194, 2008. 